\title{
The Influence of Lorentz Force on the AC Loss in sub-size Cable-in-Conduit Conductors for ITER
}

\author{
Arend Nijhuis and Herman H. J. ten Kate \\ University of Twente, Enschede, The Netherlands \\ Pierluigi Bruzzone \\ ITER JWS Naka, Japan
}

\begin{abstract}
The cable-in-conduit superconductors for the ITER coils have operating current in excess of $40 \mathrm{kA}$ and function under fast ramp conditions and fields up to $13 \mathrm{~T}$. The transverse Lorentz force acting on strands may reduce the effective contact resistance between strands in the cable and as a consequence, the coupling loss will increase. This influence is investigated with a sub-size jacketed cable having $81 \mathrm{Cr}$-coated $\mathrm{Nb}_{3} \mathrm{Sn}$ strands. The $\mathrm{AC}$ loss is measured with a sinusoidal and trapezoidal magnetic field superimposed to a stationary background field of 1 or $2 \mathrm{~T}$ while the cable carries a constant transport current up to about $30 \mathrm{kA}$. The $\mathrm{AC}$ Ioss is determined by a pick-up coil system and partly with a calorimeter for calibration purposes. The $n \cdot \tau$ at 0 current declines after cyclic loading, from $9 \mathrm{~ms}$ in the virgin state to $2 \mathrm{~ms}$ after several loads. The increase of the interstrand coupling loss due to Lorentz forces accompanied by resistance- hysteresis and relaxation effects as observed in the loss are discussed. The total loss increases considerably due to interference of transport current and induced coupling currents with rising transport current and DC field.
\end{abstract}

\section{INTRODUCTION}

The AC loss in superconductors increases when in addition to alternating external fields a stationary transport current flows [1]. Possible contributions to the increase are the transport current loss or self field loss, the enhanced interstrand coupling loss due to the Lorentz force working on the contacts in a cable, and finally the interference of screening, coupling and transport currents in the filaments.

An important parameter that determines the coupling currents between strands in superconducting cables is the interstrand resistance. Its value depends on a variety of factors, as the cable layout, compacting factor, production conditions, impregnation, temperature, transverse load, contact deformation and history. In our case the strands in the cable are subjected to a varying transverse load and elastic (and plastic) deformation caused by the local Lorentz force. This force and the manufacturing conditions of the conductors determine the ultimate interstrand coupling loss as generated by the alternating magnetic field around the cable.

As part of the ITER conductor development program, the $\mathrm{AC}$ loss in $\mathrm{Nb}_{3} \mathrm{Sn}$ (sub)cables in transverse and longitudinal $\mathrm{AC}$ fields

Manuscript received August 25 1996. These investigations are carried out as part of the contract NET/91-338 between the NET-team in Garching and the University of Twente. in combination with transport current and a DC background field have been measured in a test facility built up in Twente [2]. A study of $\mathrm{AC}$ loss in 3 types of experimental subcables in absence of a jacket was performed earlier in order to investigate the effects of the application of transverse and longitudinal fields and transport current simultaneously $[3,4]$. As a first order approach a transport current dependent $\mathrm{n} \tau$ of about $1 \mathrm{~ms} / \mathrm{kA}$ was found in a loose 27 strands (sub)cable without jacket for applied transverse fields and transport currents up to $10 \mathrm{kA}$ at $2 \mathrm{~T}$.

The Lorentz forces working on the wires in the cable vary due to any change of the field or the currents. It was reported before [5] that the resistance between two strands not only decreases when the applied force is raised but above that a hysteresis behaviour of the resistance versus applied force is noticed. Moreover a relaxation of the resistance value within a few minutes after unloading of the force was observed. This effect may cause significant hysteresis and relaxation in the interstrand coupling loss versus changing transport current [4].

The ITER full size cable is performed as a CIC conductor and is compacted to a void fraction of 0.36 . In order to investigate the effect of heavy compaction the experiment is extended to a round CIC conductor with 81 strands in a steel jacket. The average pressure on the strands under the tested conditions is approximately a factor of 2 lower than in ITER.

In this paper we focus on the influence of a stationary transport current on the AC loss of a cable in conduit conductor subjected to an applied transverse AC field.

\section{AC LOSSES}

The coupling loss time constant can be determined from the initial slope of the loss curve (energy loss per cycle versus frequency), assuming that the hysteresis loss per cycle at low excitation is independent from the frequency. The coupling loss per volume of strand, per cycle, is at low frequencies proportional to $\mathrm{f}$ and to $\mathrm{Ba}^{2}$ :

$$
\mathrm{Q}_{\mathrm{cpl}}=\nu \cdot\left(\pi / \mu_{0}\right) \cdot \mathrm{B}_{\mathrm{a}}^{2} \cdot \omega \cdot \mathrm{n} \cdot \tau \quad\left[\mathrm{J} / \mathrm{m}^{3} \cdot \mathrm{cycle}\right]
$$

The time constant $\tau[\mathrm{s}]$ is the decay time constant of the induced currents. The volume fraction of the total amount of strand volume involved in the power dissipation is expressed by v. Several dominant time constants may exist that are associated with relatively small volume fractions of a cable [6]. The shape factor is represented by $n$, which amounts to $n=2$ in wires with circular cross sections and uniformly distributed filaments and is not the same for all dominant time constants. The effective $n \tau$ value determined by 
the low frequency limit can be regarded as the summation of the contributions $\Sigma v_{k} \cdot n_{k} \cdot \tau_{k}$, for all cabling stages. The volume fraction $v_{k}$ is the fraction involved in the creation of coupling current loss represented by the time constant $\tau_{\mathrm{k}}$. This effective volume fraction is difficult to estimate for individual time constants [6]. In the following the $v \cdot n \cdot \tau$ will be indicated just by $n \tau$, because $v$ and $n$ are unknown and the $n \tau$ value is related to the total strand volume. The applied field is $B_{a} \cdot \sin (2 \pi f t)$.

When a transport current is present in a superconducting cable the hysteresis loss will decrease noticeable with increasing amplitudes of the transverse field $B_{a}$ provided the filaments are fully penetrated. For a wire a certain critical value of the current exists for a given applied alternating field, [1] at which the voltage drop along the wire rises sharply from a level corresponding to the dynamic resistance of the filaments to a level corresponding to the dynamic resistance as a whole. A similar performance may be expected in a complex cabled superconductor, resulting in enhanced losses when the transport current is raised.

\section{TEST SET UP AND CONDUCTOR}

A superconducting transformer is used to load the hairpin shaped sample of cable with current [2]. A feed back loop is applied to compensate for the decay of the current caused by the dissipation in the two joints. Transverse AC fields up to $0.5 \mathrm{~T}$ can be generated in combination with a DC background field of $2 \mathrm{~T}$. A cross section of the part where the loss is measured is drawn in Fig. 1. The magnetization loops can be measured with pick up coils and the loss is calibrated with boil off calorimetry. The loss in the stainless steel sample holder is negligible. The total length of the cable involved in the calorimetric measurement is $0.9 \mathrm{~m}$. The conductor under test is a jacketed 81 strands twisted cable $(1 \times 3 \times 3 \times 3 \times 3)$, compacted to a void fraction of 0.40 . The $\mathrm{Nb}_{3} \mathrm{Sn}$ strand with a strand diameter of $0.73 \mathrm{~mm}$, is produced by Vacuumschmelze (HNST4000) and has a $2 \mu \mathrm{m}$ thick Cr coating (plated by Duralloy).

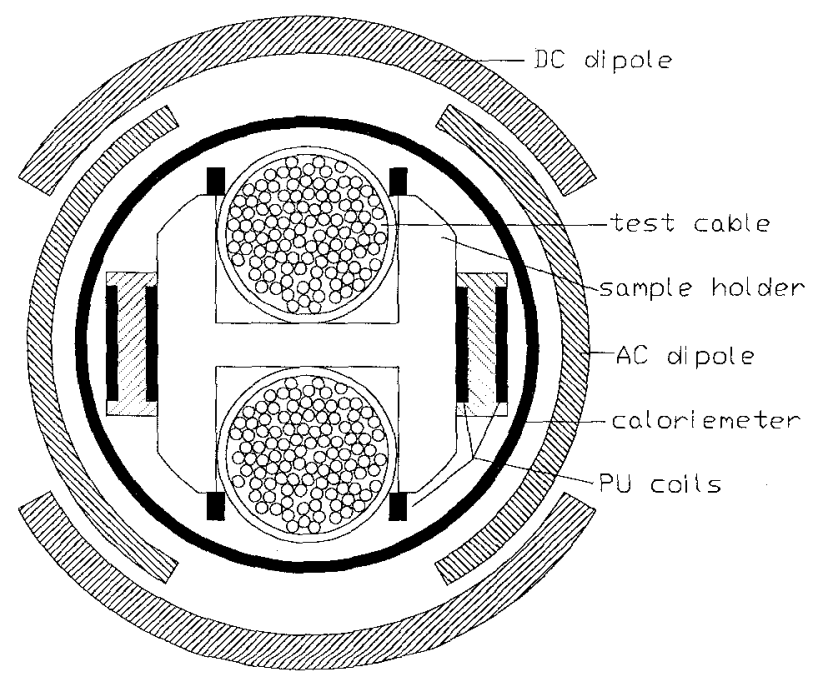

Fig. 1. Cross section of the test set up (not on real scale).

\section{AC LOSS WITHOUT TRANSPORT CURRENT}

The coupling loss time constant, appears to decrease after cycling of the load. The results gathered in Fig. 2, clearly show a decrease of $n \cdot \tau=9.3 \mathrm{~ms}$ from the virgin state to $n \cdot \tau=2 \mathrm{~ms}$ after several experiments. (Between measurement number 4 and 5 the test set up was on room temperature). It is suggested that this effect is due to an increase of the interstrand contact resistance on its turn caused by strand movements. Strand displacements may be present by Lorentz forces which are induced during the ramp of the DC field.

After several loads of the cable with transport current, the crossover strand contacts lose their engagement and the interstrand contact resistance increases. The final time constant of $n \cdot \tau=2 \mathrm{~ms}$, is about the single strand time constant. The same effect has been noticed in previous experiments in this test set up [3]. The coupling loss time constant for 27 strands unjacketed cables without load, was found to be less than $1 \mathrm{~ms}$ after several cycles.

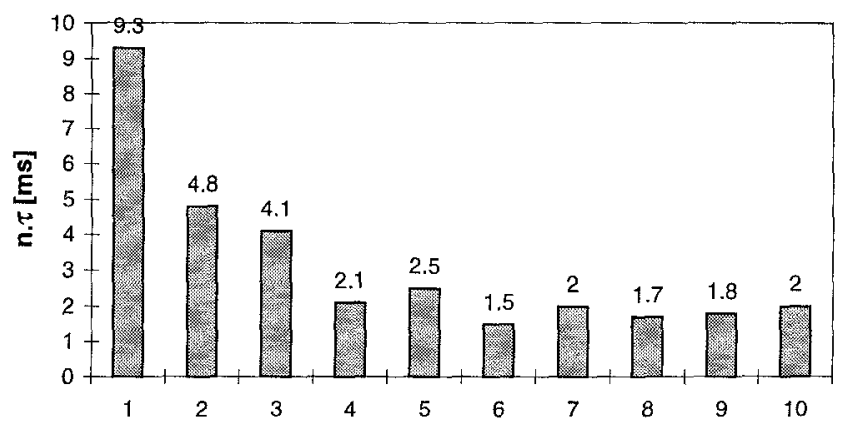

Fig. 2. The coupling loss time constant $n \cdot \tau$ versus the number of measurements in chronological sequence $\left(\mathrm{B}_{\mathrm{dc}}=2 \mathrm{~T}\right)$.

\section{AC LOSS WITH DC TRANSPORT CURRENT}

The measured quench current without applied $\mathrm{AC}$ field is about $30 \mathrm{kA}$ and the maximum stable current amounts to about $28 \mathrm{kA}$ at $1 \mathrm{~T}$. The resistance of both joints in the secondary loop, determined from the decay of the current and the calculated inductance of the loop, is less than $1 \mathrm{n} \Omega$ below a cable current of $20 \mathrm{kA}$.

The total loss $Q_{\text {tot }}$ is measured calorimetrically without and in a transverse sinusoidal AC field with an amplitude of $200 \mathrm{mT}$ at a frequency of $0.5 \mathrm{~Hz}$. The losses are taken at a stationary background fields of $B_{\mathrm{dc}}=1$ and $2 \mathrm{~T}$. The results of the loss curves thus found are presented in Fig. 3.

A clear increase of the loss with increasing transport current is observed. During the step-by-step linear increase of the current, occasionally a sudden fall in the loss is noticed of about 10 to even $30 \%$. It is suggested that this effect is originated by strand displacements, resulting in a redistribution of the current among the strands. As a consequence a more favourable situation seems to be reached with respect to the loss production. Two curves with the same label $\left(I_{T}\right.$ up, $\left.B_{d c}=2 T\right)$ are plotted to show an assessment of the reproducibility after repeated measurement of the loss curve.

Without applied AC field, loss is produced above $I_{T}=15 \mathrm{kA}$ for $B_{d c}=2 \mathrm{~T}$ increasing up to $70 \mathrm{~mW}$ just before quenching. The 


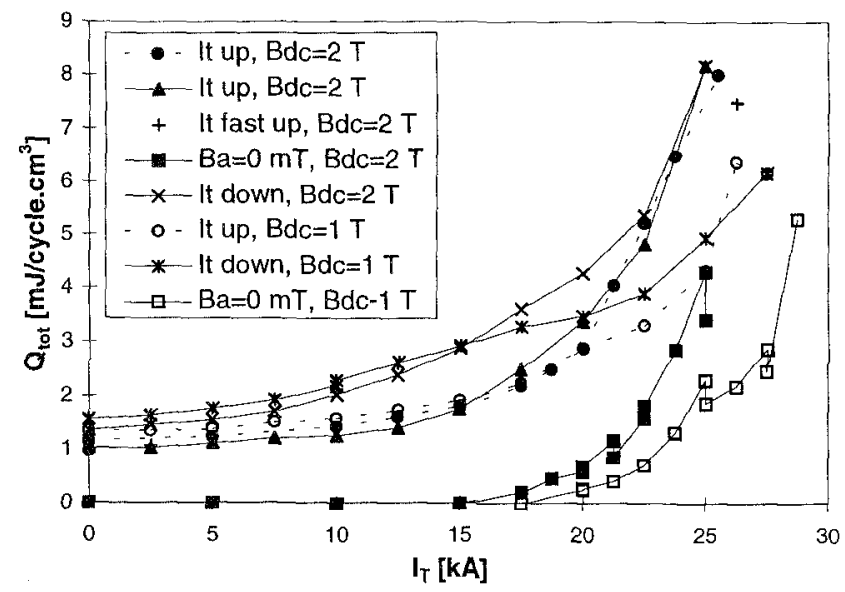

Fig. 3. Total loss (calorimetrically) versus $I_{T}$ with and without an applied $A C$ field $B a=200 \mathrm{mT}$ and $\mathrm{f}=0.5 \mathrm{~Hz}$, in background fields of $\mathrm{Bdc}=1$ and $2 \mathrm{~T}$.

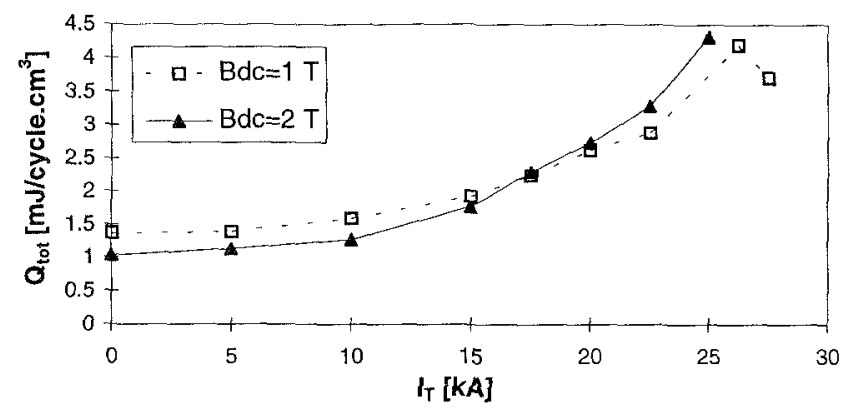

Fig. 4. Qtot (calorimetrically) with $\mathrm{Ba}=200 \mathrm{mT}$ and $\mathrm{f}=0.5 \mathrm{~Hz}$, minus Qtot with $\mathrm{Ba}=0 \mathrm{mT}$, versus $\mathbf{I}_{\mathbf{T}}$, for $\mathrm{Bdc}=1$ and $2 \mathrm{~T}$.

production of loss in the conductor for $B_{d c}=1 \mathrm{~T}$ starts above $\mathrm{I}_{\mathrm{T}}=17.5 \mathrm{kA}$, increasing up to $80 \mathrm{~mW}$ just before a quench appears. The critical current of the strand material for $\mathrm{Bdc}=2 \mathrm{~T}$ is unknown, but a conductor degradation may be a possible cause of the loss production starting at $60 \%$ of the quench current.

Parasitic heat originating from the joints are a possible cause too but this is not likely. The excellent cooling along the joints, and the distance to the calorimeter $(0.2 \mathrm{~m})$ is supposed to prevent heat conduction produced by the joints into the calorimeter. The difference between the loss production for a given current at different $B_{d c}$ and $B_{a}=0 \mathrm{mT}$ supports this assumption.

In Fig. 4 the difference between the loss with and without $\mathrm{AC}$ field is shown aiming a compensation for the DC transport current loss. The total loss convincingly increases with rising transport current and applied DC field up to a factor of 4 .

The total loss also depends on the history of the transport current as can be seen in Fig. 3. The loss with decreasing current is distinctly higher than the loss measured with increasing current.

If the transport current is switched off, still part of the initial increase is left. This remnant loss contribution decays within a few minutes after removal of the load. Not only a hysteresis but moreover a relaxation effect in the total loss per cycle of the applied AC field versus $I_{T}$ is noticed. In this context it should be mentioned that the system time constant of the calorimeter is just a few seconds due to its small gas volume and thereby fast enough to follow the actual loss versus time.

Characteristic results of the magnetisation measurements using pick-up coils, are shown in Figs. 5 and 6 in terms of the total magnetisation loss $Q_{M}$ versus frequency at different transport currents. A clear increase of the initial slope of the curves representing the inter- and intrastrand coupling loss is observed at transport currents of $I_{T} \geq 10 \mathrm{kA}$ for all amplitudes of the applied AC field and different background fields. All the coupling loss time constants are gathered in Fig. 7. It is striking that the value measured at the highest load is still smaller than the 9 ms measured in the virgin state.

The hysteresis loss decreases noticeable with increasing transport current up to $\mathrm{I}_{\mathrm{T}}>10 \mathrm{kA}$. For small values of $\mathrm{B}_{\mathrm{a}}$ and $\mathrm{I}_{\mathrm{T}}$, the transport current fits in between the saturated zones induced by the applied field change [1]. At high transport currents $I_{T} \geq 15 \mathrm{kA}$, the total magnetisation loss sharply increases. It is suggested that the

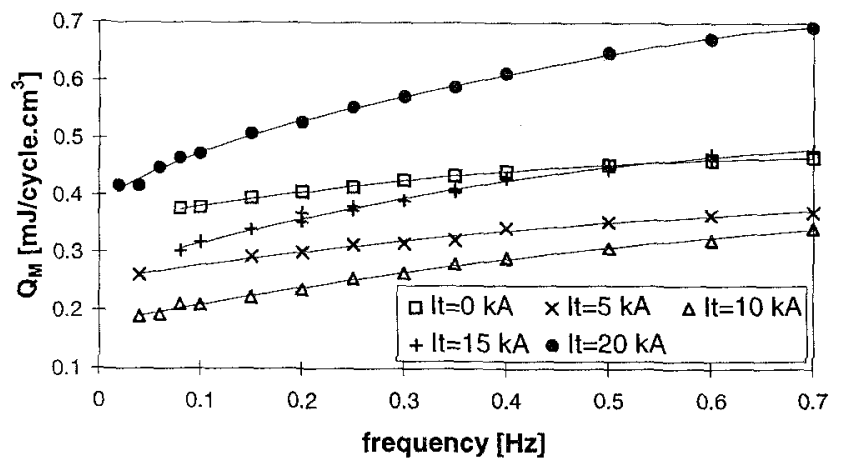

Fig. 5. Magnetisation loss versus $\mathrm{f}$ from $\mathrm{PU}$ coils. $\mathrm{Ba}=0.1 \mathrm{~T}$ and $\mathrm{Bdc}=2 \mathrm{~T}$.

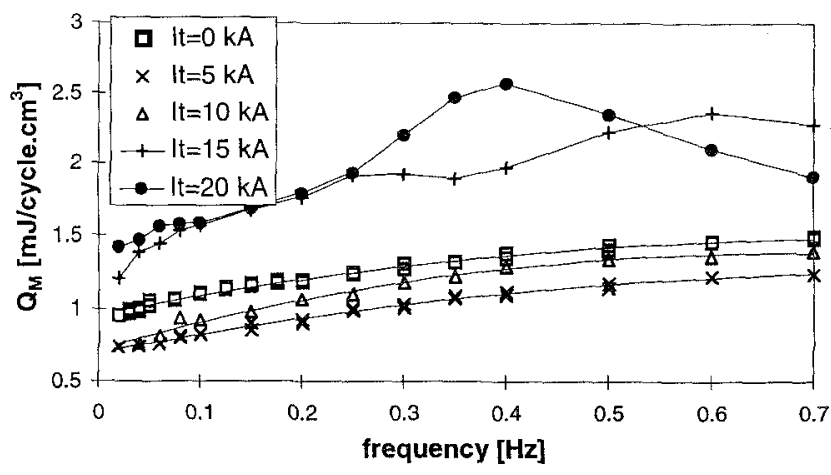

Fig. 6. Magnetisation loss versus $f$ from PU coils. $B a=0.2 T$ and $B d c=2 T$.

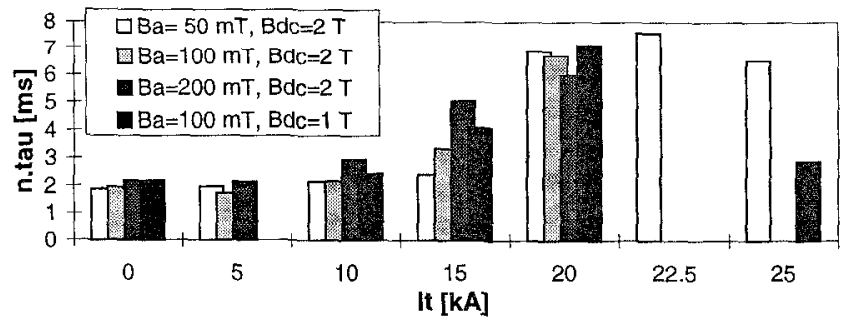

Fig. 7. Coupling loss time constants for several applied AC fields, taken from the initial slope of the loss curve at $B d c=1$ and $2 \mathrm{~T}$. 
cable is damaged and as a consequence resistive areas are created (Fig. 3, $\mathrm{Ba}=0 \mathrm{mT}$ ). The applied $\mathrm{AC}$ field causes a continuous redistribution of the transport current giving a change of flux as detected by the PU coils.

The initial slope of the $Q_{M}$ (f) curve seems to become less steep and for $B_{a}=200 \mathrm{mT}, B_{d c}=2 T$ and $I_{T} \geq 15 \mathrm{kA}$ in Fig. 6, a decrease of the magnetisation loss is noticed with rising frequency indicating saturation.

The influence of a ramped field on the coupling currents in and the redistribution of the transport current in the cable is also investigated by applying a trapezoidal field pulse. The decay of the coupling currents versus time elapsed after the pulse are monitored by the PU coil voltage. The PU voltage signals for the time interval directly after the pulse presented in Fig. 8 are low pass filtered at $\mathrm{f}=100 \mathrm{~Hz}$. The PU signals after $80 \mathrm{~ms}$ up to $220 \mathrm{~ms}$ shown in Fig. 9 are low pass filtered at $\mathrm{f}=10 \mathrm{~Hz}$. A considerable increase of coupling loss with rising transport current is clearly observed. For the time interval between 5 and $10 \mathrm{~ms}$ the decay of the voltage in the case of $\mathrm{I}_{\mathrm{T}}=25 \mathrm{kA}$ can be described using a time constant $\tau$ of about $4 \mathrm{~ms}$. The first $5 \mathrm{~ms}$ after the pulse are ignored because of the presence of higher harmonics and filtering. After $100 \mathrm{~ms}$ the PU signal can be described with high time constants progressively gaining up with transport current to more than $200 \mathrm{~ms}$, but with PU voltage amplitudes of about 25 times smaller compared to the one found for $\tau \approx 4 \mathrm{~ms}$. These high time constants for coupling currents in multistage cables related to relatively small volume fractions of

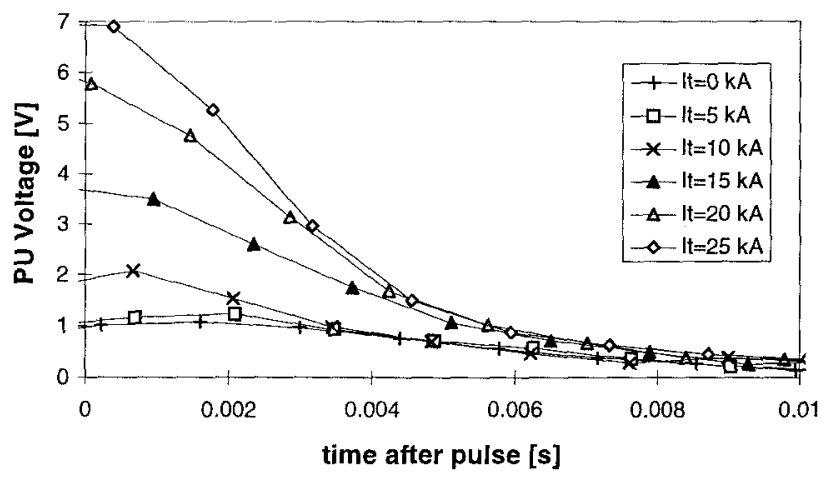

Fig.8. Decay of pick up coils voltage after pulse with $\triangle B a=175 \mathrm{mT}$ $\Delta \mathrm{t}=254 \mathrm{~ms}(\mathrm{~dB} / \mathrm{dt}=0.7 \mathrm{~T} / \mathrm{s})$ and $\mathrm{Bdc}=2 \mathrm{~T}$. Low pass filtered at $100 \mathrm{~Hz}$

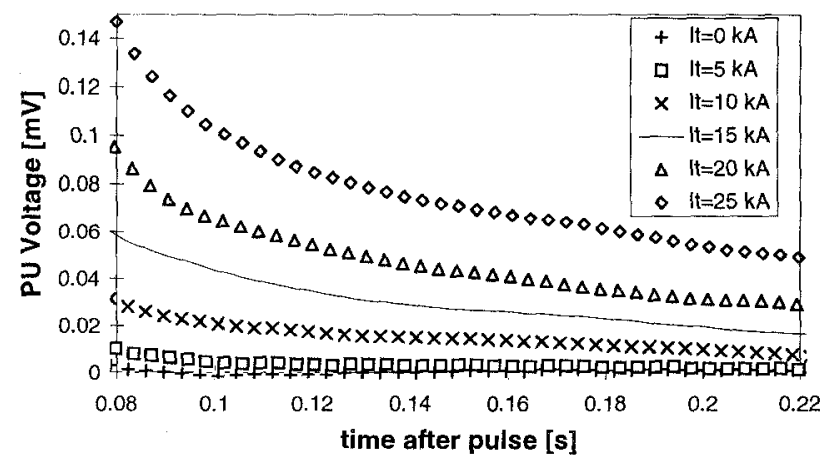

Fig. 9. Decay of pick up coils voltage after pulse with $\triangle B a=175 \mathrm{mT}$ $\Delta \mathrm{t}=254 \mathrm{~ms}(\mathrm{~dB} / \mathrm{dt}=0.7 \mathrm{~T} / \mathrm{s})$ and $\mathrm{Bdc}=2 \mathrm{~T}$. Low pass filtered at $10 \mathrm{~Hz}$. the cable are consistent with earlier experiments [6]. The effective $\mathrm{n} \tau$ value of $7 \mathrm{~ms}$ representing $\Sigma \mathrm{v}_{\mathrm{k}} \cdot \mathrm{n}_{\mathrm{k}} \cdot \tau_{\mathrm{k}}$, determined with the sinusoidal applied field at high load, is in good agreement with the dominantcoupling loss time constant $\tau \approx 4 \mathrm{~ms}$ found with the trapozoidal pulse.

Another effect possibly playing a role here are the so called boundary induced coupling currents [7] characterized in general by relatively high time constants. They are present in cables with variations of internal (resistances) or external factors (field) along the length of the cable. In our case there is a stepwise transition in contact resistance due to the joints inside the cable and the $\mathrm{AC}$ field as well as the DC field is only present at parts of the conductor.

\section{CONCLUSIONS}

The coupling loss time constant of the ITER like subcable without transport current declines from $9 \mathrm{~ms}$ in the virgin state to $2 \mathrm{~ms}$ after several electromagnetic loads.

The measured quench current in this particular cable without applied AC field is less than $30 \mathrm{kA}$ and the maximum stable current amounts to about $28 \mathrm{kA}$. A DC Transport current loss without applied AC field appears at $60 \%$ of the quench current, presumably due to damages in the cable.

A DC transport current in combination with an AC applied field causes an increase of the $\mathrm{AC}$ loss up to a factor of 4 under the measured conditions. The average pressure on the strands under ITER conditions is about 2 times higher than for the subcable in the test. The observed increase partly consists of interstrand coupling loss which is caused by a decrease of the interstrand resistance due to the Lorentz forces in the cable. The $n \tau$ value measured at the highest load is still smaller than the one measured in the virgin state.

Hysteresis in crossover resistance and relaxation are found in the interstrand coupling loss due to changing Lorentz forces.

The decay of the coupling currents after a trapezoidal pulse also shows a considerable increase of coupling loss with rising transport current confirming the results found with sinusoidal applied field.

\section{REFERENCES}

[1] T. Ogasawara et al., 'Transient field losses in multifilamentary composite conductors carrying de transport currents', Cryogenics, Vol. 20, (1980), p. 216-222.

[2] G.B.J. Mulder, H.H.J. ten Kate, A. Nijhuis and L.J.M. van de Klundert, 'A new test set up to measure the AC losses of the conductors for NET', IEEE Trans. on Magn., Vol. 27, No. 2, March 1991, p. 2190.

[3] A. Nijhuis and H.H.J. ten Kate, 'Study of the effect of transport current and combined transverse and longitudinal fields on the AC loss in NET prototype conductors.' IEEE Trans. on Magn., Vol. 30, 1994, p. 2006.

[4] A. Nijhuis and H.H.J. ten Kate, 'Interstrand coupling loss in NET prototype cabled conductors carrying a DC transport current', Applied Supercond., Vol. 1, pp.35-38, EUCAS '93, October 1993.

[5] $\mathrm{D}$ ter Avest et al., 'On the interstrand resistances in superconducting cables', Cryogenics, Vol. 30, (1990) September Supplement, p. 694.

[6] A. Nijhuis, H.H.J. ten Kate, J.L. Duchateau and P. Bruzzone, 'Coupling loss time constants full size Nb3Sn model conductor for fusion magnets', to be published in Proceedings of ICMC 1995

[7] A.P. Verweij, 'Electrodynamics of Superconducting cables in accelerator magnets', thesis, University of Twente, Enschede, September 1994. 